\title{
Cognitive outcome 5 years after bilateral chronic stimulation of subthalamic nucleus in patients with Parkinson's disease
}

\author{
M F Contarino, A Daniele, A H Sibilia, L M A Romito, A R Bentivoglio, G Gainotti, A Albanese
}

See Editorial Commentary, p 221

J Neurol Neurosurg Psychiatry 2007;78:248-252. doi: 10.1136/jnnp.2005.086660

See end of article for authors' affiliations

Correspondence to: A Daniele, Istituto di Neurologia, Università Cattolica, Largo A Gemelli, 8, I-00168 Rome, Italy; adaniele@rm.unicatt.it

Received 19 December 2005 Revised version received 12 April 2006

Accepted 24 April 2006

Published Online First

11 May 2006

\begin{abstract}
Aim: To assess the long-term cognitive and behavioural outcome after bilateral deep brain stimulation (DBS) of the subthalamic nucleus (STN) in patients affected by Parkinson's disease, with a 5-year follow-up after surgery.

Methods: 11 patients with Parkinson's disease treated by bilateral DBS of STN underwent cognitive and behavioural assessments before implantation, and 1 and 5 years after surgery. Postoperative cognitive assessments were carried out with stimulators turned on.

Results: A year after surgery, there was a marginally significant decline on a letter verbal fluency task $(p=0.045)$ and a significant improvement on Mini-Mental State Examination $(p=0.009)$. 5 years after surgery, a significant decline was observed on a letter verbal fluency task $(p=0.007)$ and an abstract reasoning task $(p=0.009)$, namely Raven's Progressive Matrices 1947. No significant postoperative change was observed on other cognitive variables. No patient developed dementia 5 years after surgery. A few days after the implantation, two patients developed transient manic symptoms with hypersexuality and one patient developed persistent apathy.

Conclusion: The decline of verbal fluency observed 5 years after implantation for DBS in STN did not have a clinically meaningful effect on daily living activities in our patients with Parkinson's disease. As no patient developed global cognitive deterioration in our sample, these findings suggest that DBS of STN is associated with a low cognitive and behavioural morbidity over a 5-year follow-up, when selection criteria for neurosurgery are strict.
\end{abstract}

The long-term cognitive and behavioural effects of bilateral STN DBS were investigated in 70 patients with Parkinson's disease followed up for 3 years. ${ }^{11}$ In this study, a decline of verbal fluency, an improvement of depression and an increased apathy were observed 3 years after surgery. Some patients showed behavioural changes (aggressive behaviour, hypomania, depression and psychosis), which were mostly transient. Recently, the long-term outcome of bilateral DBS of STN was investigated in a multicentre study conducted in 49 patients with Parkinson's disease followed up for 3 or 4 years. ${ }^{16}$ This study showed that stimulation of the STN induced a significant improvement in Parkinsonian motor symptoms and activities of daily living 3-4 years after surgery. Among the adverse events, the authors reported memory decline or psychiatric disturbances (including hallucinations, delirium, depression, apathy and anxiety), which occurred in about $30 \%$ of the patients.

In two recent investigations, the long-term outcome of bilateral DBS of STN was investigated in patients with a 5year follow-up. ${ }^{17}{ }^{18}$ In one study conducted on 49 patients with Parkinson's disease, ${ }^{17}$ cognitive performance was assessed by means of the Mattis Dementia Rating Scale (MDRS) ${ }^{19}$ and a frontal-lobe score. ${ }^{4}$ Five years after surgery, there was a marked improvement of both motor function, while off drugs, and activities of daily living, a statistical trend towards a decline on the MDRS (reflecting the appearance of progressive dementia in three patients between the third and the fifth postoperative years) and a significant decline in the average frontal-lobe

Abbreviations: DBS, deep brain stimulation; LEDD, levodopa-equivalent daily dose; MDRS, Mattis Dementia Rating Scale; MMSE, Mini-Mental State Examination; MWCST, Modified Wisconsin Card Sorting Test; RAVLT, Rey's Auditory Verbal Learning Test; RPM, Raven's Progressive Matrices; STN, subthalamic nucleus; UPDRS, Unified Parkinson's Disease Rating Scale reported cases of depression ${ }^{10}$ or increased apathy, ${ }^{11}$ whereas cases of mania were described in other studies ${ }^{12-14}$ and an improvement of depression ${ }^{1}$ or apathy ${ }^{15}$ was also found. 
score. Another study carried out on 37 patients with Parkinson's disease ${ }^{18}$ also assessed cognitive performance by means of MDRS $^{19}$ and a frontal score. ${ }^{20}$ Five years after the implantation, there was an improvement in Parkinsonian motor symptoms and activities of daily living and a reduction of levodopa-related motor complications and levodopa daily doses. However, a significant decline in cognitive performance was detected on the MDRS and the frontal score.

To our knowledge, no extensive neuropsychological data have been reported so far in patients with a follow-up $>3$ years. The aim of the present study was to assess the long-term cognitive and behavioural outcome after bilateral DBS of the STN in a series of patients followed up for 5 years after surgery.

\section{METHODS \\ Patients}

From our initial series of 26 patients with Parkinson's disease who underwent bilateral DBS of STN, 11 patients completed 5year follow-up cognitive and behavioural assessments after implantation. All patients who were selected for implantation showed disabling motor fluctuations and on-state dyskinesias, according to previously described inclusion criteria. ${ }^{21}$ Severe psychiatric symptoms, moderate or severe cognitive impairment, as measured by scores $<20$ on the Mini-Mental State Examination (MMSE), and diagnosis of dementia were among the exclusion criteria. ${ }^{6}$ Table 1 shows the main demographic and clinical characteristics of patients with Parkinson's disease at the time of implantation.

\section{Procedures}

All patients received a bilateral stereotactic STN implant, according to previously described neurosurgical procedures. ${ }^{21}$ Patients underwent cognitive assessments while they were on drugs for Parkinson's disease, both preoperatively and postoperatively. Cognitive assessment was carried out by means of a neuropsychological test battery described previously, ${ }^{6}$ including the MMSE, tasks of verbal (digit span forward and backward) and spatial (Corsi's block-tapping test) short-term memory, tasks of episodic verbal memory (Rey's Auditory Verbal Learning Test (RAVLT)) and non-verbal abstract reasoning (Raven's Progressive Matrices (RPM) 1947), a letter verbal fluency task and a task assessing frontal-executive cognitive functions (MWCST). In addition, a clinical interview was conducted to detect the presence of behavioural abnormalities or psychiatric disorders. A preoperative assessment (baseline) was carried out during the 4 weeks preceding the bilateral implantation of electrodes. Postoperative assessments were carried out 12 and 60 months after implantation, with stimulators turned on.

\section{Statistical analysis}

To assess the outcome, the scores obtained on each postoperative assessment were compared with preoperative scores by means of the Wilcoxon's signed-rank test, given the small sample size and non-normally distributed data. Given the

Table 1 Main demographical and clinical characteristics of patients with Parkinson's disease

\begin{tabular}{ll}
\hline Number of patients & 11 \\
\hline Sex (M:F) & $7: 4$ \\
Age at implant (years; mean (SD)) & $57.5(7.4)$ \\
Disease duration at implant (years; mean (SD)) & $15.5(5.6)$ \\
Age at 5-year follow-up (years; mean (SD)) & $62.6(7.5)$ \\
Educational level (years; mean (SD)) & $11.0(5.1)$ \\
\hline
\end{tabular}

$F$, female; $M$, male explorative nature of our study, the standard non-corrected significance $\alpha$ level of $p<0.05$ was used, to reduce the risk of a type II error. However, given the risk of type I error due to multiple comparisons, significant results should be considered cautiously when the $\alpha$ levels are only marginally $<0.05 .{ }^{8}$ To evaluate postoperative changes among individual patients on cognitive variables, each individual postoperative raw score was transformed to a standard z score, using means and standard deviations (SD) of the sample at baseline. The criterion of an increase $>1$ SD was used to tally individual postoperative improvements, whereas the criterion of a decrease $>-1$ SD was used to tally individual postoperative declines. ${ }^{6}$

\section{RESULTS}

\section{Group outcome}

Five years after surgery, parkinsonian motor symptoms improved in all patients with Parkinson's disease. As compared with preoperative assessment (table 2), there was a significant improvement in Unified Parkinson's Disease Rating Scale (UPDRS) motor scores $(\mathrm{p}=0.003)$ and activities of daily living $(p=0.003)$ on 12-month and 60-month postoperative assessments without drugs for Parkinson's disease and a significant reduction in the total levodopa-equivalent daily dose (LEDD) on 12-month $(p=0.006)$ and 60-month $(p=0.005)$ postoperative assessments.

Table 3 shows the group outcome on cognitive variables.

One year after surgery, there was a marginally significant postoperative decline on the letter verbal fluency task (mean decline: $-16.2 \% ; p=0.045)$ and a significant postoperative improvement on the MMSE (mean improvement: $+8.9 \%$; $\mathrm{p}=0.009)$. Five years after surgery, there was a significant postoperative decline on the letter verbal fluency task (mean decline: $-33.1 \% ; \mathrm{p}=0.007$ ) and on RPM (mean decline: $-10.1 \%$; $p=0.009$ ). Moreover, there was a trend towards a postoperative decline on both the delayed recall of the RAVLT (mean decline $-15.8 \% ; \mathrm{p}=0.075$ ) and on Corsi's span backward (mean decline $-17.4 \% ; \mathrm{p}=0.091$ ), whereas no significant postoperative change was observed on other cognitive variables. When the raw scores obtained by each individual patient on the letter verbal fluency task at 5 years were adjusted for age and educational level, ${ }^{22}$ only 3 of 11 patients performed below the normal range. In these three patients, the scores on the verbal fluency task were 12.2, 10.6 and 10.2, respectively, with a cutoff score of 17.35. Interestingly, two of these three patients performed below the normal range on the verbal fluency task also before the implantation. Similarly, when the raw scores obtained by each individual on the RPM at 5 years were adjusted for age and educational level, ${ }^{22}$ only 2 of 11 patients performed slightly below the normal range (their scores were 18.3 and 18.0, respectively, with a cut-off score of 18.96). In one of these two patients, the score on RPM was below the normal range even on preoperative assessment. No patient developed dementia 5 years after surgery, according to the criteria of the Diagnostic and statistical manual of mental disorders, 4 th edn. ${ }^{23}$

Comparison between scores obtained on cognitive tasks 1 year versus 5 years after surgery showed a significant longterm decline on the letter verbal fluency task (mean decline $-20.1 \%$; $p=0.005$ ), the MMSE (mean decline $-7.2 \%$; $\mathrm{p}=0.008$ ) and the Corsi's span backward (mean decline $-19.1 \% ; \mathrm{p}=0.028$ ).

Table 4 shows the individual outcome on cognitive variables on 1-year and 5-year postoperative assessments.

One year after surgery, on some cognitive variables (verbal fluency, digit span forward, perseverative errors on the MWCST) the percentage of patients who declined was clearly higher than the percentage of patients who improved, whereas on other tasks (MMSE, digit span backward) the percentage of 
Table 2 Motor scores, activities of daily living, levodopa-equivalent daily dose (LEDD) at baseline and at 1-year and 5-year followup, with stimulators turned on, on and off drugs

\begin{tabular}{|c|c|c|c|c|c|c|}
\hline & Baseline off & Baseline on & 1 year off & 1 year on & 5 years off & 5 years on \\
\hline \multicolumn{7}{|l|}{ UPDRS-III } \\
\hline Mean (SD) & $57.73(10.65)$ & $24.27(8.68)$ & $27.82(15.33)$ & $20.82(12.11)$ & $22.00(10.31)$ & $18.10(11.39)$ \\
\hline Variation $v$ baseline (\%) & & & -51.8 & -14.2 & -61.9 & -25.4 \\
\hline $\mathrm{p}$ Value & & & 0.003 & 0.374 & 0.003 & 0.284 \\
\hline \multicolumn{7}{|l|}{ UPDRS-II } \\
\hline Mean (SD) & $32.36(5.41)$ & $10.27(9.68)$ & $8.73(5.02)$ & 5.91 (4.99) & $8.82(6.23)$ & $7.09(6.06)$ \\
\hline Variation $v$ baseline (\%) & & & -73.0 & -42.5 & -72.8 & -31.0 \\
\hline $\mathrm{p}$ Value & & & 0.003 & 0.126 & 0.003 & 0.398 \\
\hline \multicolumn{7}{|l|}{ LEDD } \\
\hline Mean (SD) & $1406.55(750.15)$ & & 427.66 (354.89) & & $489.80(394.46)$ & \\
\hline Variation $v$ baseline (\%) & & & -69.6 & & -65.2 & \\
\hline $\mathrm{p}$ Value & & & 0.006 & & 0.005 & \\
\hline
\end{tabular}

patients who improved was substantially higher than the percentage of patients who declined. Five years after surgery, on the verbal fluency task there was a remarkably high percentage of patients who declined, although $46 \%$ of the patients remained stable. At 5 years, on tasks of abstract reasoning (RPM), episodic memory (delayed recall of RAVLT) and short-term memory (digit span forward and Corsi's span backward), the percentage of patients who declined was substantially higher than the percentage of those who improved. A marked individual variability was observed on digit span forward at 5 years. A minority of patients showed no postoperative change on this verbal short-term memory task, whereas most patients had either a postoperative improvement or decline.

Over a 5-year follow-up, 3 of 11 patients experienced adverse events. A few days after the implantation, two patients, who were previously described elsewhere, ${ }^{12}$ developed transient manic symptoms with hypersexuality, which lasted for some months and then gradually disappeared spontaneously. Moreover, one patient developed persistent apathy. None of our patients committed suicide or expressed suicidal intentions.

\section{DISCUSSION}

To our knowledge, this is the first study that extensively reports the long-term cognitive effects in patients with Parkinson's disease 5 years after lead implantation in the STN.

The results of neuropsychological assessment in our 11 patients treated by STN DBS with 5 years of follow-up show a significant decline in the letter verbal fluency task and in the abstract reasoning task (RPM). However, when the raw scores obtained by each patient 5 years after surgery were adjusted for age and educational level, only 3 of 11 patients performed below the range of normal subjects on the verbal fluency task, whereas on RPM only 2 of 11 patients performed slightly below the normal range. Interestingly, most of the patients who performed below the normal range on the verbal fluency task and RPM 5 years after surgery did perform below the normal range on these two tasks preoperatively also. One year after surgery, we observed a marginal decline in the letter verbal fluency task and a significant postoperative improvement on the MMSE, which could most likely reflect a practice effect. ${ }^{6}$ The analysis of postoperative cognitive changes in individual patients 5 years after surgery showed some degree of interindividual variability in cognitive outcome. Although on the verbal fluency task $56 \%$ of patients declined, $46 \%$ of the patients remained stable. At 5 years, the percentage of patients who declined on tasks of abstract reasoning, episodic memory and short-term memory was substantially higher than the percentage of those who improved.

Our observation of a decline in verbal fluency 5 years after implantation for STN DBS is in agreement with previous studies reporting a decreased verbal fluency in patients with a 1-year ${ }^{146}$ or a 3-year follow-up. ${ }^{11}$ The only two studies published so far reporting a 5 -year follow-up after bilateral DBS of STN ${ }^{17}{ }^{18}$ were mainly focused on the long-term outcome of Parkinsonian motor symptoms. In both investigations, a significant decline in cognitive performance was detected on the MDRS and on neuropsychological measures sensitive to

Table 3 Results obtained on cognitive variables in the overall group of 11 patients at baseline, 1-year and 5-year follow-up

\begin{tabular}{|c|c|c|c|c|c|c|c|}
\hline Variable & $\begin{array}{l}\text { Score } \\
\text { range }\end{array}$ & $\begin{array}{l}\text { Baseline } \\
\text { Mean (SD) }\end{array}$ & $\begin{array}{l}1 \text { year } \\
\text { Mean (SD) }\end{array}$ & $\begin{array}{l}\text { Comparison } 1 \text { year } \\
v \text { baseline } \\
\% \text { (p Value) }\end{array}$ & $\begin{array}{l}5 \text { years } \\
\text { Mean (SD) }\end{array}$ & $\begin{array}{l}\text { Comparison } 5 \text { years } \\
\text { v baseline } \\
\% \text { (p Value) }\end{array}$ & $\begin{array}{l}\text { Comparison } 5 \text { years } \\
\text { v } 1 \text { year } \\
\% \text { (p Value) }\end{array}$ \\
\hline MMSE* & $0-30$ & $26.5(3.0)$ & $28.9(1.2)$ & $+8.9 \%(0.009)$ & $26.8(2.0)$ & $+1.0(0.760)$ & $-7.2(0.008)$ \\
\hline $\begin{array}{l}\text { Raven Progressive } \\
\text { Matrices } 1947^{*}\end{array}$ & $0-36$ & $28.5(5.2)$ & $28.2(6.0)$ & $-1(0.541)$ & $25.5(6.2)$ & $-10.2(0.009)$ & $-9.4(0.050)$ \\
\hline Rey immediate recall* & $0-75$ & 40.5 (12.9) & $39.8(13.1)$ & $-1.6(0.959)$ & $37.8(12.6)$ & $-6.5(0.721)$ & $-5.0(0.477)$ \\
\hline Rey delayed recall* & $0-15$ & $9.2(4.0)$ & $8.6(4.8)$ & $-5.9(0.418)$ & $7.7(3.7)$ & $-15.8(0.075)$ & $-10.5(0.214)$ \\
\hline Digit span forward* & & $5.5(1.1)$ & $5.4(0.8)$ & $-3.3(0.584)$ & $5.1(1.1)$ & $-8.2(0.176)$ & $-5.1(0.311)$ \\
\hline Digit span backward* & & $3.7(1.0)$ & $4.2(1.5)$ & $+12.2(0.161)$ & $3.7(1.7)$ & $0.0(0.933)$ & $-10.9(0.176)$ \\
\hline Corsi's span forward* & & $4.8(1.1)$ & $4.9(0.8)$ & $+1.9(0.715)$ & $4.6(1.1)$ & $-3.8(0.554)$ & $-5.6(0.273)$ \\
\hline Corsi's span backward* & & $4.2(1.3)$ & $4.3(1.1)$ & $+2.2(0.787)$ & $3.5(1.0)$ & $-17.4(0.091)$ & $-19.1(0.028)$ \\
\hline Letter verbal fluency* & & $33.0(12.6)$ & $27.6(9.4)$ & $-16.2(0.045)$ & $22.1(9.5)$ & $-33.1(0.007)$ & $-20.1(0.005)$ \\
\hline MWCST: correct criteria* & *0-6 & $4.3(1.9)$ & $4.5(1.8)$ & $+4.9(0.201)$ & $4.2(1.9)$ & $-3.5(0.715)$ & $-8.0(0.612)$ \\
\hline MWCST: total errors $\dagger$ & $0-48$ & $12.6(12.1)$ & $10.8(11.9)$ & $-13.8(0.327)$ & $14.2(9.7)$ & $+13.0(0.779)$ & $+31.1(0.062)$ \\
\hline $\begin{array}{l}\text { MWCST: perseverative } \\
\text { errors } t\end{array}$ & $0-48$ & $5.0(6.3)$ & $4.6(6.5)$ & $-7.3(0.499)$ & $4.5(4.9)$ & $-9.1(0.554)$ & $-2(1.000)$ \\
\hline
\end{tabular}

Significant data are in bold face. ${ }^{*}+$, improvement; - , decline; lower score $=$ worse performance. $\dagger-$, improvement; + , decline; lower score $=$ better clinica condition. MMSE, Mini-Mental State Examination; MWCST, Modified Wisconsin Card Sorting Test. 
Table 4 Individual postoperative changes on cognitive variables at 1-year and 5-year follow-up

\begin{tabular}{|c|c|c|c|c|c|c|}
\hline \multirow[b]{2}{*}{ Variable } & \multicolumn{2}{|c|}{ Improvement } & \multicolumn{2}{|l|}{ No change } & \multicolumn{2}{|l|}{ Decline } \\
\hline & 1 year $(\%)$ & 5 years $(\%)$ & 1 year $(\%)$ & 5 years $(\%)$ & 1 year $(\%)$ & 5 years $(\%)$ \\
\hline MMSE* & 45 & 9 & 55 & 82 & 0 & 9 \\
\hline Raven Progressive Matrices 1947* & 18 & 0 & 64 & 73 & 18 & 27 \\
\hline Rey immediate recall* & 18 & 9 & 64 & 73 & 18 & 18 \\
\hline Rey delayed recall* & 27 & 9 & 46 & 55 & 27 & 36 \\
\hline Digit span forward* & 0 & 18 & 82 & 46 & 18 & 36 \\
\hline Digit span backward* & 27 & 18 & 73 & 55 & 0 & 27 \\
\hline Corsi's span forward* & 18 & 18 & 73 & 55 & 9 & 27 \\
\hline Corsi's span backward* & 9 & 0 & 82 & 82 & 9 & 18 \\
\hline Letter verbal fluency* & 0 & 0 & 82 & 46 & 18 & 54 \\
\hline MWCST: correct criteria* & 0 & 11 & 89 & 67 & 11 & 22 \\
\hline MWCST: total errors $†$ & 33 & 0 & 45 & 78 & 22 & 22 \\
\hline MWCST: perseverative errors $\dagger$ & 0 & 0 & 78 & 89 & 22 & 11 \\
\hline
\end{tabular}

MMSE, Mini-Mental State Examination; MWCST, Modified Wisconsin Card Sorting Test.

${ }^{*}$ An increase $>1$ SD indicates improvement, whereas a decrease $>-1$ SD indicates decline. $†$ An increase $>1$ SD indicates decline, whereas a decrease $>-1$ SD indicates improvement.

frontal-lobe dysfunction (frontal-lobe scores) 5 years after implantation, but no information about performance on verbal fluency tasks was available, as no extensive neuropsychological data have been reported in such studies.

In our sample, behavioural adverse events included transient mania with hypersexuality in two patients, which appeared a few days after surgery, did not require treatment and might be due to the synergistic effects of the implantantion (surgical procedure or STN stimulation) with other factors such as dopaminergic treatment or individual predisposition. ${ }^{12}$

At 5 years, the postoperative decline of verbal fluency did not seem to have a clinically meaningful effect on daily living activities in our patients with Parkinson's disease. As the decline in verbal fluency observed after implantation for STN DBS seems to begin immediately after surgery, it has been speculated that such decline could be due to surgical microlesions possibly involving cortical-basal ganglionic circuits, which might play a critical role in word retrieval processes, ${ }^{24}$ rather than due to STN stimulation in itself. ${ }^{25}$ Alternatively, it has been suggested that STN stimulation might give rise to a decreased activation of inferior frontal, insular and temporal cortex in the left cerebral hemisphere, resulting in a decreased verbal fluency. ${ }^{26}$ Poor performance on verbal fluency tasks is frequently observed in patients with Parkinson's disease who are not treated by neurosurgery ${ }^{27}$ and in those treated by pallidotomy, particularly after left-sided pallidotomy. ${ }^{24}{ }^{28}$ These findings should be taken into account in studies reporting a verbal fluency decline in patients treated by chronic stimulation of STN.

A limitation of our study is the lack of a control group of patients with Parkinson's disease who did not undergo neurosurgical intervention, as cognitive decline may occur over time in patients with the disease, owing to the disease progression. Several other studies on DBS in Parkinson's disease have the same limitation, owing to the difficulties in the recruitment of a control group of patients with Parkinson's disease who are not treated by neurosurgical procedures, which should be ideally matched at baseline to the group of patients who undergo DBS of STN with regard to several variables (age, educational level, disease duration, severity of motor impairment and overall cognitive status). Moreover, longitudinal studies focused on an extensive neuropsychological assessment are lacking in patients with Parkinson's disease, even in patients who do not undergo neurosurgical procedures. Such longitudinal studies are also needed, to compare the long-term cognitive outcome in patients with Parkinson's disease who are not treated by neurosurgery with those of patients who undergo STN DBS.
In conclusion, our findings suggest that DBS of STN in patients with Parkinson's disease may be associated with low cognitive and behavioural morbidity over a 5-year follow-up and can therefore be considered relatively safe as to the longterm outcome, when appropriate criteria are used to select candidates for neurosurgery. Further studies are needed to elucidate the mechanisms underlying the postoperative decline of verbal fluency observed in patients with Parkinson's disease treated by DBS of STN, which is already detectable in these patients early after STN DBS and becomes more evident in late postoperative stages.

\section{Authors' affiliations}

M F Contarino*, A Daniele*, A H Sibilia, L M A Romito, A R Bentivoglio, G Gainotti, A Álbanese, Istituto di Neurologia, Università Cattolica del Sacro Cuore - Largo Gemelli, Rome, Italy

*These authors contributed equally to this work.

Competing interests: None.

\section{REFERENCES}

1 Ardouin C, Pillon B, Peiffer E, et al. Bilateral subthalamic or pallidal stimulation for Parkinson's disease affects neither memory nor executive functions: a consecutive series of 62 patients. Ann Neurol 1999;46:217-23.

2 Trepanier LL, Kumar R, Lozano AM, et al. Neuropsychological outcome of GPi pallidotomy and GPi or STN deep brain stimulation in Parkinson's disease. Brain Cogn 2000;42:324-47.

3 Saint-Cyr JA, Trepanier LL, Kumar R, et al. Neuropsychological consequences of chronic bilateral stimulation of the subthalamic nucleus in Parkinson's disease. Brain 2000;123:2091-108.

4 Pillon B, Ardouin C, Damier P, et al. Neuropsychological changes between "off' and "on" STN or GPi stimulation in Parkinson's disease. Neurology 2000;55:411-18.

5 Jahanshahi M, Ardouin CM, Brown RG, et al. The impact of deep brain stimulation on executive function in Parkinson's disease. Brain 2000;123: 11 142-5.

6 Daniele A, Albanese A, Contarino MF, et al. Cognitive and behavioural effects of chronic stimulation of the subthalamic nucleus in patients with Parkinson's disease. J Neurol Neurosurg Psychiatry 2003;74:175-82.

7 Witt K, Pulkowski U, Herzog J, et al. Deep brain stimulation of the subthalamic nucleus improves cognitive flexibility but impairs response inhibition in Parkinson disease. Arch Neurol 2004:61:697-700.

8 Alegret $M$, Junque $C$, Valldeoriola $F$, et al. Effects of bilateral subthalamic stimulation on cognitive function in Parkinson disease. Arch Neurol 2001;58:1223-7.

9 Schroeder U, Kuehler A, Haslinger B, et al. Subthalamic nucleus stimulation affects striato-anterior cingulate cortex circuit in a response conflict task: a PET study. Brain 2002; 125:1995-2004.

10 Doshi PK, Chhaya N, Bhatt MH. Depression leading to attempted suicide after bilateral subthalamic nucleus stimulation for Parkinson's disease. Mov Disord 2002;17:1084-5. 
11 Funkiewiez $\mathbf{A}$, Ardouin $\mathrm{C}$ Caputo $\mathrm{E}$, et al. Long term effects of bilateral subthalamic nucleus stimulation on cognitive function, mood, and behaviour in Parkinson's disease. J Neurol Neurosurg Psychiatry 2004;75:834-9.

12 Romito LM, Raja M, Daniele A, et al. Transient mania with hypersexuality after surgery for high frequency stimulation of the subthalamic nucleus in Parkinson's disease. Mov Disord 2002;17:1371-4.

13 Kulisevski J, Berthier ML, Gironell A, et al. Mania following deep brain stimulation for Parkinson's disease. Neurology 2002;59:1421-4.

14 Herzog J, Reiff J, Krack P, et al. Manic episode with psychotic symptoms induced by subthalamic nucleus stimulation in a patient with Parkinson's disease. Mov Disord 2003;18:1382-4.

15 Czernecki V, Pillon B, Houeto JL, et al. Does bilateral stimulation of the subthalamic nucleus aggravate apathy in Parkinson's disease? J Neurol Neurosurg Psychiatry 2005;76:775-9.

16 Rodriguez-Oroz MC, Obeso JA, Lang AE, et al. Bilateral deep brain stimulation in Parkinson's disease: a multicentre study with 4 years follow-up. Brain 2005; 128:2240-9.

17 Krack P, Batir A, Van Blercom N, et al. Five-year follow-up of bilateral stimulation of the subthalamic nucleus in advanced Parkinson's disease. N Engl J Med 2003:349:1925-34.

18 Schüpbach WMM, Chastan N, Welter ML, et al. Stimulation of the subthalamic nucleus in Parkinson's disease: a 5 year follow up. J Neurol Neurosurg Psychiatry 2005:76:1640-4

19 Schmidt R, Freidl W, Fazekas F, et al. The Mattis Dementia Rating Scale: normative data from 1,001 healthy volunteers. Neurology 1994;44:964-6.
20 Pillon B, Dubois B, Lhermitte F, et al. Heterogeneity of cognitive impairment in progressive supranuclear palsy, Parkinson's disease and Alzheimer's disease. Neurology 1986;36:1179-85.

21 Moro E, Scerrati M, Romito LM, et al. Chronic subthalamic nucleus stimulation reduces medication requirements in Parkinson's disease. Neurology 1999:53:85-90.

22 Carlesimo GA, Caltagirone C, Gainotti G. The Mental Deterioration Battery: normative data, diagnostic reliability and qualitative analyses of cognitive impairment. The Group for the Standardization of the Mental Deterioration Battery. Eur Neurol 1996:36:378-84.

23 American Psychiatric Association. Diagnostic and statistical manual of mental disorders. 4th edn. Washington: American Psychiatric Association, 1994.

24 Troster Al, Woods SP, Fields JA. Verbal fluency declines after pallidotomy: an interaction between task and lesion laterality. Appl Neuropsychol 2003;10:69-75.

25 Alegret $M$, Valldeoriola F, Marti M, et al. Comparative cognitive effects of bilateral subthalamic stimulation and subcutaneous continuous infusion of apomorphine in Parkinson's disease. Mov Disord 2004;19:1463-9.

26 Schroeder U, Kuehler A, Lange KW, et al. Subthalamic nucleus stimulation affects a frontotemporal network: a PET study. Ann Neurol 2003;54:445-50.

27 Matison R, Mayeux R, Rosen J, et al. "Tip of the tongue" phenomenon in Parkinson's disease. Neurology 1982;32:567-70.

28 Schmand B de Bie RM, Koning-Haanstra M, et al. Unilateral pallidotomy in PD: a controlled study of cognitive and behavioral effects. The Netherlands Pallidotomy Study (NEPAS) group. Neurology 2000;54:1058-64.

\section{BNF for Children 2006, second annual edition}

In a single resource:

- guidance on drug management of common childhood conditions

- hands-on information on prescribing, monitoring and administering medicines to children

- comprehensive guidance covering neonates to adolescents

For more information please go to bnfc.org 